\title{
Standardtheorie und invertierte Standardtheorie
}

Das erste steht uns frei, beim zpeiten sind wir Knechte.

1. Gegeben seien die folgenden zwei Sätze aus dem Papyrus Westcar:

(1) iy. $n=i$ ? $r$ nis $r=k \ldots$

"Ich bin hierher gekommen, um dich (im Auftrag meines Vaters Cheops) zu rufen.“ (pWestcar 7, 20)

(2) $m=k$ wi $\boldsymbol{i y} \cdot k w$

„Siehe, ich bin gekommen." (pWestcar 8, 12)

In der deutschen Übersetzung, einer durchaus korrekten, und, sieht man einmal von „siehe“ als einem altväterlichen Sprachgebrauch der Bibelübersetzungen ab, einer durchaus natürlichen Wiedergabe des Ägyptischen, - in der deutschen Übersetzung also zeigen die beiden Sätze eine und dieselbe Verbalform: „Ich bin gekommen. “ Im Ägyptischen dagegen stehen zwei verschiedene Verbalformen:

- Satz (1) zeigt ein sč̣m. $n=f:$ iy. $n=i$,

- Satz (2) dagegen zeigt das Pseudopartizip: iy.kw.

Es sind dies zwei Sätze in der Art der Sätze, an Hand derer Hans Jacob Polotsky in seinen berühmten „Egyptian Tenses“ von 1965 den entscheidenden zweiten Schritt in der Entwicklung seiner Theorie der ägyptischen Verbalsyntax tat ${ }^{1}$.

Wissenschaftsgeschichtlich war, grob gesagt, der Gang der Dinge dieser: 1944 hatte Polotsky in seinen nicht weniger berühmten „Études de syntaxe copte“, ausgehend vom Koptischen, Sätze des Typs (1) erklärt - zum mindesten ansatzweise ${ }^{2} .1965$, in den „Tenses“, fügte er dann, auf dem Ansatz der „Études“ aufbauend, die Erklärung der Sätze des Typs (2) hinzu. Aus diesem Zweischritt ergab sich mit einiger Konsequenz das, was man heute als „Standardtheorie“ der ägyptischen Syntax oder genauer: Verbalsyntax bezeichnet ${ }^{3}$.

Wählt man, gerade umgekehrt wie Polotsky dies tat, als ersten Einstieg Satz (2) und expandiert einen auf dieser Basis gewonnenen Theoriekern auf die Erklärung des Satzes (1), so gewinnt man, ebenfalls mit einiger Konsequenz, eine Alternativ-Theorie, die man entsprechend der Umkehrung der Erklärungsfolge als ,invertierte Standardtheorie“ bezeichnen könnte, eine

* Der Text geht auf einen Vortrag zurück, der am 13. 1. 1995 im Institut für Sudanarchäologie und Ägyptologie der Humboldt-Universität, Berlin, gehalten wurde. Weitere Zwischenversionen wurden für eine Vorlesung an der Universität Tübingen im Sommersemester 1995 und für ein Seminar an der University of California, Los Angeles, im Oktober 1996 ausgearbeitet.

H. J. Polotsky, Egyptian Tenses, The Israel Academy of Sciences and Humanities, Proceedings II 5, Jerusalem 1965 (im folgenden zitiert als „Tenses").

2 H. J. Polotsky, Études de syntaxe copte, Kairo 1944, bes. S. 19-98 (Nachdruck: id., Collected Papers, Jerusalem 1971 , S. $102-207$, bes. S. $125-202$ ).

${ }^{3}$ Zum Einzelnen s. L. Depuydt, The Standard Theory of the Emphatic Forms in Classical (Middle) Egyptian: A Historical Survey, in: OLP 14 (1983), S. 13-54; W. Schenkel, Einführung in die altägyptische Sprachwissenschaft, Darmstadt 1990, S. 145-158. 
Theorie, die ansatzweise in Verf.s „Tübinger Einführung“ von 1994 (schwarzer Umschlag) ${ }^{4}$ entwickelt und in der Version von 1997 (lila Umschlag) ( $^{5}$ weiter ausgebaut wurde.

In welcher Weise sich aus der unterschiedlichen Reihenfolge der Erklärung der beiden Sätze (1) und (2) die unterschiedlichen Theorien ergeben, soll im folgenden vorgeführt werden.

2. Was vorgeführt wird, ist nicht der Gang der Dinge, wie er sich wissenschaftsgeschichtlich zutrug, der Gang der Dinge in seiner ganzen Verschlungenheit, mit all seinen Um- und Seitenwegen, mit all den Meinungen und Gegenmeinungen, die in der Gemeinschaft der Forschenden geäußert wurden ${ }^{6}$. Was vorgeführt wird, ist vielmehr eine theoretische Nachkonstruktion der Aufdeckungsprozedur, ein Weg, den man als den kürzesten hätte einschlagen können, wenn man von vornherein gewußt hätte, auf welches Ziel man zusteuern soll, eine Nachkonstruktion aus unserem heutigen, besseren Verständnis der Sachverhalte, einem besseren Verständnis, das sich als Folge überhaupt erst des Ganges der Dinge eingestellt hat.

Hier also die theoretische Nachkonstruktion eines Weges, der ohne Umwege zur „Standardtheorie“ führt, eine Nachkonstruktion, die, wie gesagt, von der Erklärung des Satzes (1) ausgeht:

(1) iy. $n=i \mathrm{C} r$ niśr $r=k \ldots$

„Ich bin hierher gekommen, um dich (im Auftrag meines Vaters Cheops) zu rufen.“

In einem solchen Satz - das ist die entscheidende erste Polotskysche Einsicht - liegt der Akzent auf einer Adverbialen, hier auf dem adverbialen Ausdruck Präposition plus Infinitiv $r$ niś $r=k$,um dich zu rufen“:

(1) „Um dich zu rúfen (und aus keinem anderen Grund), bin ich hierher gekommen.“

oder, in einer holprigen, dennoch, weil zweckmäßig, sehr beliebten Philologenübersetzung: „Daß ich hierher gekommen bin, ist, um dich zu rúfen.“

Nicht das Kommen ist also das wesentliche Neue der Aussage, sondern der Zweck des Kommens. Im Zusammenhang des zitierten Textes, einer der Erzählungen des Papyrus Westcar, erklärt der Prinz Hardedef dem Wundermann Dedi den Grund seines Kommens - um ihn zu Pharao zu rufen -, nicht das Faktum seines Kommens, das in der Begrüßungssituation selbstevident ist.

Grammatikalisch realisiert ist die Begründung als ein präpositionaler Ausdruck aus Präposition plus Infinitiv : $r$ nis $r=k$, um dich zu rufen“. Nun kann man - freilich muß man nicht einen Satz, der das wesentliche Neue als adverbialen Ausdruck formuliert, als den altbekannten Satztyp Adverbialsatz interpretieren, in dem ebenfalls das wesentliche Neue in einem adverbialen Ausdruck liegt, im adverbialen Prädikat. So wie man versteht

(3) $h r . t=k m p r(. w)=k$

„Dein Bedarf (ist) in deinem Haus.“" (Bauer B 1, 124/neu),

so kann man auch Satz (1) verstehen als

(1) iy. $n=i$ r $r$ nis $r=k$

„Daß ich hierher gekommen bin, (ist,) um dich zu rufen.“

So wie im Adverbialsatz das wesentliche Neue als adverbiales Prädikat steht, so stellt auch in Satz (1) der adverbiale Ausdruck, der das wesentliche Neue ausdrückt, das Prädikat des Satzes dar. So wie im Adverbialsatz das Subjekt ein Substantiv ist - $h r . t=k$, dein Bedarf“ - so liegt

\footnotetext{
${ }^{4}$ W. Schenkel, Tübinger Einführung in die klassisch-ägyptische Sprache und Schrift, Tübingen: Wolfgang Schenkel 1994 (im folgenden zitiert als ,Tübinger Einführung, Version 1994").

5 W. Schenkel, Tübinger Einführung in die klassisch-ägyptische Sprache und Schrift, Tübingen: Wolfgang Schenkel 1997 (im folgenden zitiert als ,Tübinger Einführung, Version 1997`).

${ }^{6}$ Vgl. hierzu W. Schenkel, op. cit. (s. oben Anm. 3), S. 145-158 (mit Literaturhinweisen).
} 
dann auch in Satz (1) ein substantivisches Subjekt vor: iy. $n=i$ 3 „Das Kommen meinerseits hierher" oder, immer noch holprig und doch etwas eleganter, „Daß ich hierher gekommen bin“. Dieser Analogieschluß, die Projektion der Subjekts-Prädikats-Struktur des Adverbialsatzes in Satz (1), ob zwingend oder ob nur möglich, war eine wissenschaftsgeschichtlich entscheidende Weichenstellung.

\section{Anmerkung:}

Aus dieser frühen Setzung ergab sich im Zuge der Ausgestaltung der Standardtheorie eine Erklärungstendenz, die man als Prädikatisierungsstrategie bezeichnen könnte. Es ist dies der Versuch, möglichst jede Haupt-Betonung eines Satzes als Prädikat zu verstehen. Betroffen von dieser Strategie sind u. a. fokussierte Satzteile, mit der Folge, daß, was pragmatisch veranlaßt ist, der kontrastive Akzent, in die syntaktische Ebene verlagert wird. Auf diese Weise wurde namentlich, was die invertierte Standardtheorie - und nicht erst diese - für ein betontes Subjekt hält, in der in-Konstruktion und im fokussierenden Substantivalsatz zum Prädikat erklärt. Dem soll hier nicht im einzelnen nachgegangen werden. Zur Veranschaulichung können die unterschiedlichen Darstellungen in der „Tübinger Einführung " dienen, deren Version von 1991 (roter Umschlag) ${ }^{7}$ einen standardtheoretischen Standpunkt vertritt, deren Versionen von 1994 (schwarzer Umschlag) ${ }^{8}$ und von 1997 (ila Umschlag) ${ }^{9}$ dagegen einen nicht-standardtheoretischen beziehen. Ein anderer Effekt dieser Erklärungsstrategie ist die Tendenz, Interrogativa als Prädikat anzusetzen, auch in anderen Sätzen als denen vom Typ des Satzes (1). Auch diesem Befund soll hier nicht im einzelnen nachgegangen werden.

Zurück zur Erklärung von Satz (1): Die Verbalform iy. $n=i$ ist ein Substantiv und zugleich eine finite, nach der Person flektierte Verbalform. Sie ist, kurz gesagt, eine substantivische (finite) Verbalform.

3. Die substantivischen Verbalformen bilden nach der Standardtheorie ein dreistufiges „Tempus"system, das im Aktiv bei einem intransitiven Verb der Bewegung etwa so aussieht (der größeren Deutlichkeit halber sei statt iyi ,,kommen“ das Verbum pri , herausgehen“ gewählt):

\begin{tabular}{l|l|l} 
„Tempus“ & substantivisch & adverbial \\
\hline Perfekt & $\operatorname{pr} . n=f$ & $\operatorname{pr}(. w)$ (Pseudop.) \\
\hline Generalis (Aorist) & $\operatorname{prr}=f$ & $\operatorname{pr}=f$ \\
\hline Futur (Prospektiv) & $\operatorname{pr}(. y)=f$ & $\operatorname{pr}(. y)=f$
\end{tabular}

Charakteristisch für die substantivischen Verbalformen ist die geminierte Form im Generalis (Aorist): $p r r=f$,daß er (üblicherweise) herausgeht" (bei Verben, die geminieren können) und das sç̌m. $n=f$ bei intransitiven Verben der Bewegung: $p r . n=f$,daß er herausging“, Verben, die das Perfekt sonst mit dem Pseudopartizip bilden, wie das in Satz (2) der Fall ist:

(2) $m=k$ wi iy. $k w$

„Siehe, ich bin gekommen.“

So weit zur Erklärung des Satzes (1) als erstem Schritt in der Entwicklung der Standardtheorie.

4. Bevor von hier aus zur Erklärung des Satzes (2) weitergeschritten wird, seien kurz Sätze betrachtet, die weder zum Typ des Satzes (1) noch zum Typ des Satzes (2) gehören. Es handelt sich um Sätze wie

Tübinger Einführung in die klassisch-ägyptische Sprache und Schrift, Tübingen: Wolfgang Schenkel 1991 (im folgenden zitiert als ,Tübinger Einführung, Version 1991“), 』8.3 bzw. \6.1.

8 Tübinger Einführung, Version 1994, \ 8.5 .3 bzw. $\int 6.1$.

Tübinger Einführung, Version 1997, § 8.5.2 bzw. \6.1. 
(5) $m r r=f-i r r f$

„Wenn er will, so tut er.“ (Pyr. \412b)

(belegt als Satzwort: ein ,Will-er-so-tut-er.")

Hier steht ein $m r r=f$, eine substantivische Verbalform, ohne daß eine Adverbiale folgt. Gleiches gilt erst recht für $i r r=f$, auf das überhaupt nichts mehr folgt, geschweige denn eine Adverbiale.

Solche Sätze können als Substantivalsätze erklärt werden. Sie sind vergleichbar mit einem Satz wie

(6) ph.ti=i - ph.ti Rw

„Meine Kraft ist die Kraft des Ru.“ (CT III 341d S1C),

ein Satz, von dem sich Satz (5) nur dadurch unterscheidet, daß er in Subjekts- und Prädikatsfunktion statt gewöhnlicher Substantive substantivische Verbalformen enthält:

(5) $m r r=f-i r r=f$

„Daß-er-will ist/bedeutet/kommt gleich Daß-er-tut.“

Eine substantivische Verbalform - $m r r=f-$ steht als Subjekt, eine zweite substantivische Verbalform - irr $=f$ - steht als Prädikat.

Solche Substantivalsätze sind symmetrisch ausgebildet: Subjekt und Prädikat stehen in einem engen Wechselspiel zueinander, formal wie inhaltlich. Man nennt solche Sätze daher „Wechselsätze“, englisch „balanced sentences“.

Diese Sätze waren einmal von Alan H. Gardiner als ein Einwand gegen die Polotskysche Erklärung von Sätzen des Typs (1) vorgebracht worden ${ }^{10}$. Sie erwiesen sich indes auf die Länge der Zeit ganz im Gegenteil als eine Stütze des Ansatzes, von dem Augenblick an nämlich, als Polotsky der glückliche Gedanke kam, die Sätze als Wechselsätze zu erklären, als mehr oder minder symmetrisch gebaute Sätze ${ }^{11}$.

Von diesen Sätzen ist hier zu sprechen, weil sie, wissenschaftsgeschichtlich gesehen, genau an der Stelle ins Spiel kamen, an der sich in diesem Moment die theoretische Nachkonstruktion befindet: nach der Erklärung der Sätze vom Typ des Satzes (1) und vor der Erklärung der Sätze vom Typ des Satzes (2), auf die bald näher einzugehen sein wird. Zugleich wird der Satztyp im Hinblick auf spätere Zwecke eingeführt: Die Balance wird zum Problem werden, damit aber der Satztyp, folglich dann aber auch der Satztyp als Stütze des Ansatzes substantivischer (finiter) Verbalformen.

So weit der Einschub.

5. Noch einmal, zum letzten Mal, sei bei den Sätzen vom Typ des Satzes (1) angesetzt. Hier ist noch ein Detail zu ergänzen, das für die weiteren Überlegungen als Angelpunkt dient: In solchen Sätzen kann anstelle der Adverbialen auch eine Verbalform stehen, z. B. im folgenden Satz:

(7) $m 33 . t w=f, \boldsymbol{h} 3$ f $\boldsymbol{r}^{\boldsymbol{\prime}}-\boldsymbol{p \boldsymbol { c }} . \boldsymbol{t}$

„Daß er gesehen wird, (ist,) indem er die Bogentruppe angreift." (Sin. B 52f.)

Die zweite Verbalform, $h 3=f$,,er greift an“, ist, da sie in der adverbialen Prädikatsposition steht, eine adverbiale (finite) Verbalform, eine Form wie sie das Paradigma (4) in der rechten Spalte für das „Tempus“ Generalis (Aorist) ausweist, eine Form, die im Gegensatz zu der in

${ }^{10}$ A. H. Gardiner, Besprechung von H. J. Polotsky, Études de syntaxe copte, in: JEA 33 (1947), S. 95-101, bes. S. 99 .

i1 H. J. Polotsky, Ägyptische Verbalformen und ihre Vokalisation, in: Orientalia 33 (1964), S. 267-285, bes. S. 281 f. (Nachdruck: id., Collected Papers, Jerusalem 1971, S. $52-70$, bes. S. 66 f.). 
der linken Spalte stehenden substantivischen Verbalform bei einem Verb ult. inf. wie pri „herausgehen“ nicht geminiert.

Was das Verbalparadigma (4) angeht, gibt es also substantivische Verbalformen - solche die in der Subjektsposition des Adverbialsatzes stehen können (linke Spalte) - und adverbiale Verbalformen - solche, die in der Prädikatsposition des Adverbialsatzes stehen können (rechte Spalte). Letztere sind z. B. - so im Paradigma - am Pseudopartizip für das Perfekt der intransitiven Verben der Bewegung und an den nicht-geminierten Formen für Generalis eines Verbs ult. inf. wie pri , ,herausgehen“ festzumachen.

So weit die abschließende Ergänzung zur Erklärung der Sätze vom Typ des Satzes (1).

6. Nun, auf der Erklärung des Satzes (1) aufbauend, die Erklärung des Satzes (2) - wissenschaftsgeschichtlich gesehen die Leistung der Polotskyschen „Tenses“ -:

(2) $m=k$ wi iy. $k w$

„Siehe, ich bin gekommen."

Im Zusammenhang des zitierten Textes, derselben Erzählung des Papyrus Westcar, aus der auch Satz (1) stammt, - im Zusammenhang des Textes liegt der Akzent auf dem Kommen. Auf die Frage Pharaos „Wie kommt es, daß du dich noch nie hast blicken lassen?" antwortet der Wundermann Dedi: „Nur wer gerufen wird, kommt. Man hat mich gerufen. Also (so könnte man hier vielleicht das altväterliche ,siehe“ wiedergeben) - Also bin ich gekommen."

Die Verbalform des Satzes (2), das Pseudopartizip iy. $k w$, ist eine der gerade ergänzend besprochenen Verbalformen - siehe das Paradigma (4) -, die in der Prädikatsposition der Sätze des Typs (1) stehen können, also als adverbiale Verbalformen nachgewiesen sind. Nun der naheliegende Schluß: Wenn eine Verbalform in Sätzen vom Typ des Satzes (1) eine adverbiale Verbalform ist, dann ist eine solche Verbalform auch in Sätzen des Typs von Satz (2) eine adverbiale Verbalform.

Nicht ganz so klar ist indes die Beurteilung des Satzstücks, das in Satz (2) der Verbalform $i y . k w$ vorangeht. Im Beispielsatz und in allen Sätzen, die in diesem Schritt der Theoriebildung, wissenschaftsgeschichtlich gesehen, herangezogen wurden, stehen zwingend Partikeln wie $m=k$ oder, um eher noch wichtigere zu nennen, $i w$ oder ${ }^{\top}{ }^{\ulcorner} . n$. Dagegen gehört ein auf die Partikel folgendes Personalpronomen wie das in Satz (2) stehende Personalpronomen wi "mich, ich“ nicht zur unter allen Umständen notwendigen Ausrüstung des Satztyps. Infolgedessen stellt sich die Frage, was die Partikeln im Satzganzen darstellen. Eine Polotskysche Lösung ist die, die Partikel und was direkt zu ihr gehört, als eine Art Hauptsatz zu verstehen - im Beispielsatz der Hauptsatz $m=k$ wi ,siehe mich“ -, die folgende adverbiale Verbalform als in diesen Satz als eine Art Nebensatz - eingebettete Adverbiale ${ }^{12}$. Der ganze Satz ist dann etwa zu verstehen als:

(2) $m=k$ wi iy. $k w$

„Siehe mich, nachdem ich gekommen bin.“

Eine andere Lösung, die aber wenig Anklang fand, hat Friedrich Junge vorgeschlagen, auf dessen Extremstandpunkt später noch ( $(14$ f.) die Sprache kommen wird.

So viel zur Erklärung des Satzes (2).

7. Nun zur Alternative, der invertierten Standardtheorie, die man sich als von Satz (2) ausgehend und danach erst Satz (1) erklärend vorstellen kann:

(2) $m=k$ wi iy. $k w$

„Siehe, ich bin gekommen.“

${ }^{12}$ Tenses, $\int 41$, Ende (die Partikel $i w$ betreffend). 
Hier liegt das wesentliche Neue der Aussage, wie gesagt, in der Verbalform iy.kw ,ich bin gekommen“". Was liegt intuitiv näher, wenn man nicht, wie bei der Standardtheorie, durch die vorangehende Interpretation von Satz (1) belastet ist, - was liegt intuitiv näher, als diese finite Verbalform als verbales Prädikat zu verstehen, als prädikative Verbalform, und den ganzen Satz als Verbalsatz, den guten, alten Verbalsatz der älteren Grammatiker des Ägyptischen. Was der Verbalform vorangeht, sind vor allem Partikeln, hier etwa $m=k$, die für die Konstitution des Satzes selbst peripher sind.

Wie nun gelangt man von hier aus zur Erklärung des Satzes (1), für die auch im Rahmen der Alternativtheorie die Polotskysche Beobachtung gelten muß, daß eine Adverbiale, $r$ niś $r=k \ldots$ „um dich zu rufen ...", das wesentliche Neue der Aussage ist:

(1) iy. $n=i \Im r$ nisis $r=k \ldots$

„Um dich zu rúfen (und aus keinem anderen Grund), bin ich hierher gekommen."

Der Weg zum Ziel führt über eine genauere Analyse der peripheren Elemente, mit denen Sätze des Typs von Satz (2) vor der Verbalform erweitert werden können.

8. Die Alternativtheorie interpretiert Sätze als aus einem Satzkern und einer - in Grenzen variablen Anzahl vorderer Erweiterungen bestehend. Die gerade behandelte Erweiterung bzw., genauer betrachtet, die beiden Erweiterungen, die im Mustersatz

(2) $m=k$ wi iy. $k w$

„Siehe, ich bin gekommen."

vorliegen, $m=k$,siehe“ und wi „mich", sind solche Erweiterungen. Aber es gibt noch andere. Insgesamt hat man in etwa mit den folgenden Typen von Erweiterungen zu rechnen $^{13}$ :

I. Negationen, wie namentlich die Negation $n^{14}, \mathrm{z}$. B.:

(8) $n: r h=i s w$

„Ich habe ihn nicht kennengelernt", „Ich kenne ihn nicht.“ (Sin. B. 114)

\section{Extraponiertes Subjekts-Substantiv ${ }^{15}$ und}

III. Situierende Partikel wie $i w^{16}$, beides, II. und III., z. B. in:

(9) iw: $\boldsymbol{w} s \grave{t} \cdot \boldsymbol{w}: w \breve{b} b=f$

„Es ist der Fall: Der Angesprochene: Er antwortet",

„Der Angesprochene pflegt zu antworten.“ (Bauer B 1, 247/neu)

IV. Extraponiertes Substantiv (nicht nur Subjekt) (substantivisches, satzkonstituentenwertiges Topic) ${ }^{17}$, z. B. Objekt:

(10) $t 3=n: p h=n$ św

„Unser Land: Wir haben es erreicht.“ (Schiffbr. 11)

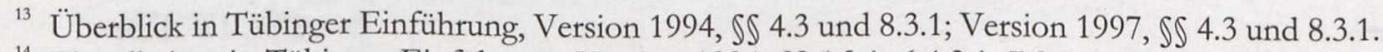

${ }^{14}$ Einzelheiten in Tübinger Einführung, Version 1994, $\iiint_{4.3 .1}, 6.4 .2 .1,7.3 .3$ und 7.3.5; Version 1997, $\$ \int ~ 4.3 .1$, 4.5.1, 6.4.2.1, 7.3.3 und 7.3.5.

${ }_{15}$ Einzelheiten in Tübinger Einführung, Version 1994, $\int \$$ 4.3.2 und 8.3.2; Version 1997, $\iint ~ 4.3 .2$ und 8.3.2.

${ }^{16}$ Einzelheiten in Tübinger Einführung, Version 1994, $\iint 4.3 .3,6.4 .2 .2,7.3 .6$ und 8.3.2; Version 1997, $\int \oint$ 4.3.3, 4.5.2, 6.4.2.2, 7.3.6 und 8.3.2.

Einzelheiten in Tübinger Einführung, Version 1994, $\iint$ 4.3.4 und 9.2.1; Version 1997, $\int \S ~ 4.3 .4$ und 9.2.1. 
V. Verbales Topic (satzwertiges Topic) ${ }^{18}$, z. B.:

(11) $r m m=s ́ n: ~ i w=f h r$ șçm

„Weinen sie: (So) hört er." (Merikare C 5, 7 - iw=f h̆ sḉm sei hier als eine einheitliche, zusammengesetzte Verbalform stehen gelassen und nicht weiter in sich selbst analysiert.)

V'. Gelegentlich beginnt die Erweiterung auch mit Präposition (nebensatzwertiges Topic), z. B.:

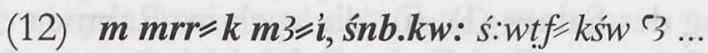

„Wenn du mich gesund zu sehen wünschst: Halte ihn hier hin ..." (Bauer B 1, 109f./neu)

VI. ir-Einleitung, abgesehen von der expliziten Markierung durch eine einleitende Partikel, meistens ir "was angeht ${ }^{\text {( }}$, , inhaltlich den beiden vorgenannten Erweiterungen, IV. und V. mit der gelegentlichen Variante $V$ '. entsprechend:

- IV entsprechend z. B.:

(13) ir ir.ti=f $n n \boldsymbol{c} \not t .\{w\} y=i:$ wnn=i $m s\}$ śnb $=f$

„Was den angeht, der tun wird, was ich gesagt habe (?): (So) werde ich der Schutz seiner Gesundheit sein. (Tübingen 458, 16 f.)

(14) ir hym $n b$ r' pn: $n$ : 'k.n=f

„Was jeden angeht, der diesen Spruch nicht kennt: (So) kann er nicht eintreten.“

(Urk. V 95, 5f.)

- V entsprechend z. B.:

(15) ir śçm $=k n n$ çt. $n=i n=k$ : wnn șhr $r=k n b r-h 3 . t$

„Wenn du auf das hörst, was ich dir gesagt habe: (So) wird jeder deiner Pläne gelingen." (Ptahh. 507f.)

- V' entsprechend (selten) z. B.:

(16) ir $\boldsymbol{m}$ wn=i $\boldsymbol{m} \underline{\mathbf{h}} \boldsymbol{r t ̦}$ : $w n=i \mathrm{~m}$ smr ...

„Als ich ein Kind war: War ich ein $\$ m r$...“ (Hatnub 22, 2f.)

Es können aber nach ir auch beliebige andere, „zitierte“ sprachliche Einheiten stehen, so etwa ein kompletter, evtl. selbst wieder erweiterter Satz ${ }^{20}$, z. B.:

(17) ir iw hr $r=f$ čms. $w$ : țšr irt.w $h r=f p w$...

„Was angeht ,Sein Gesicht ist gerötet': (So) bedeutet dies, daß die Farbe seines Gesichts rot ist ..." (pSmith 3, 20f.)

VII. Relationierende Partikel wie iśč „indessen, etc. ${ }^{\text {“621 }}$, z. B.:

(18) ist (ir=f): sb.n hm=fmśr $r T 3-T m h i$

„Indessen (aber): Seine Majestät hatte eine Truppe zum Libyerland geschickt.“ (Sin. R $11 \mathrm{f}$.)

Die diversen Erweiterungen werden als Ränge angesprochen, z. B. also die Erweiterung V, das verbale Topic, als „Rang-V-Erweiterung“. Die Benennung als Ränge besagt, daß die Erweiterungen in bestimmter Reihenfolge dem Satzkern vorgeschaltet werden. Für den gegenwärtigen

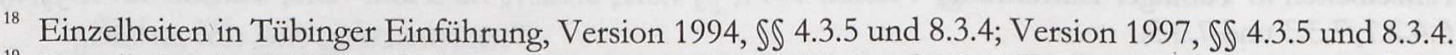

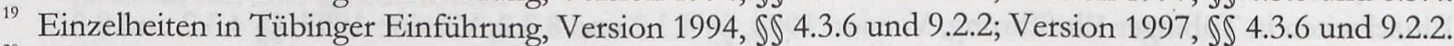

${ }^{20}$ S. W. Westendorf, Zur Doppelfunktion des jr der Hervorhebung, in: GM 151 (1996), S. 109-111; K. Jansen-Winkeln, Zitierform und Kontextform, in: GM 154 (1996), S. 45-48, bes. S. 45 f.

${ }^{21}$ Einzelheiten in Tübinger Einführung, Version 1997, \$\$ 4.7.2.2, 7.3.6.3, c) und 9.2.3. 
Zweck, den Vergleich der Standardtheorie mit der invertierten Standardtheorie, spielt diese Reihenfolge keine wesentliche Rolle.

Über die genannten Erweiterungen hinaus gibt es noch die „rangfreie“, illokutive Satzerweiterung, vorzugsweise realisiert durch $m=k$ etc. „siehe“, mit deren Hilfe eine Aussage dem Angesprochenen ,präsentiert" wird $^{22}$. Diese Erweiterung steht - syntaktisch gesehen - häufig anstelle einer situierenden Partikel (Rang III). Z. B. lautet ein Satz wie

(2) $m=k:$ wi: iy. $k w$

„Siehe, ich bin gekommen.",

wenn man das illokutive $m=k$ entfernt, z. B.

(19) *iw: $i: i y . k w$

„Es ist der Fall: Ich bin gekommen.“.

Insofern war es im Zusammenhang einer rein syntaktischen Fragestellung nicht ganz falsch, einen Satz wie Satz (2) mit einer illokutiven Partikel als Paradigmasatz zu wählen. Im gegebenen Fall steht $m=k$ in einer Doppelfunktion: pragmatisch gesehen für sich selbst, syntaktisch gesehen für eine Rang-III-Partikel wie $i w$.

Mit diesem groben Überblick über die Satzkern-Erweiterungen kann es fürs erste sein Bewenden haben. Im Augenblick ist das vordringliche Ziel, nachdem Satz (2) für die Alternativtheorie als Ausgangspunkt gesetzt und erklärt wurde, von hier aus zu einer Alternativerklärung von Satz (1) zu kommen.

9. Eine solche Alternativerklärung ist in der Tat möglich oder zum mindesten: Es ist möglich zu sagen, wie der Satztyp sprachhistorisch zustande gekommen ist. Konkret stellt sich für den Beispielsatz (1) die Lösung so dar ${ }^{23}$ : Auszugehen hat man von einem Satz mit Rang-VErweiterung, einem verbalen, satzwertigen Topic:

(1) *iy. $n=i{ }^{c} 3: i y . k w, r$ niś $r=k$

„Wenn ich hierher kam: (So) kam ich, um dich zu rufen.“

Da das zweite $i y i$, ,kommen“ inhaltlich redundant ist, wird es elidiert:

(1) iy. $n=i$ 3: $0, r$ niś $r=k$

„Wenn ich hierher kam: (so,) um dich zu rufen."

Was vom Satzkern noch übrig bleibt, ist somit nur noch die Adverbiale, die allein dadurch, daß sie der einzige verbleibende Teil des Satzkerns ist, in den Vordergrund tritt, herausgestellt wird. Das Verfahren ist im übrigen in ordentlicher deutscher Übersetzung nachvollziehbar:

(1) iy. $n=i$ 3: $0, r$ niś $r=k$

„Bin ich hierher gekommen: dann, um dich zu rufen.“

Wird die elidierte Verbalform negiert, so steht an ihrer Stelle - dem deutschen „dann“ oder „So" vergleichbar - die Partikel is als eine Art Platzhalter, der anzeigt, worauf sich die Negation bezieht:

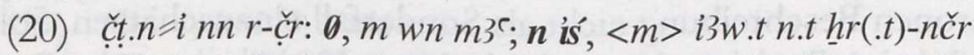

„Habe ich dies (jetzt) alles gesagt, (so) offen und ehrlich, so nicht als ,Nekropolenamt." (Moralla V 2f.)

(21) $\boldsymbol{n} \check{s} m . n=\check{c}: 0$, is' $m(w) . t .(t) i$

„Bist du weggegangen, (so,) indem du nicht tot bist.“

(CT VI 380e-f)

${ }^{22}$ Einzelheiten in Tübinger Einführung, Version 1994, $\iint 4.3 .7,6.4 .2 .3$, 7.3.6.3, a)-b) und 9.3.2; Version 1997, $\left.\left.\iint 4.3 .7,6.4 .2 .3,7.3 .6 .3, \mathrm{a}\right)-\mathrm{b}\right)$ und 9.2.4.

${ }^{23}$ Vgl. Tübinger Einführung, Version 1997, \S 4.6.1 und 8.5.3. 
(Die Negation selbst wird, wie Satz (21) zeigt, vor die real vorhandene positive Verbalform gezogen, sofern die elidierte negierte Verbalform im unmittelbar folgenden Satzkern folgt; folgt dagegen zuerst ein nicht negierter Satzkern und danach erst der Satzkern mit der elidierten negierten Verbalform, so bleibt, wie Satz (20) zeigt, die Negation vor dem Platzhalter des Verbs im zweiten Satzkern stehen.)

\section{Anmerkung:}

Dementsprechend sollte man iś auch in anderen negierten Sätzen, zum mindesten genetisch (oder tiefenstrukturell), als Platzhalter erklären können. Betroffen sind namentlich die in-Konstruktion und der Substantivalsatz. Z. B. könnte eine Fokussierungskonstruktion wie $n$ ink iśs čt "Nicht ich bin es, der gesagt hat.“" (CT VI 251i Sq3C) zurück-

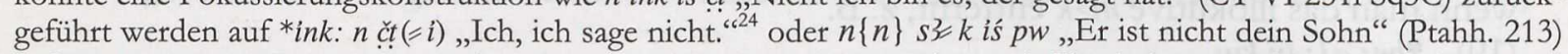
auf *s $3=k: n$ is $p w$ „Dein Sohn, nicht ist er ein solcher" (auch hier schon mit Platzhalter iś) oder schließlich $n$ ntk iś $s(. i)$ „Du bist nicht ein Mann.“ auf *ntk: $n$ ntk $s(. i)$ „Du, (du bist) nicht ein Mann.“ (Leb. 31, mit Pronomen als Platzhalter). D. h. es handelt sich ursprünglich um eine Topikalisierungskonstruktion. Details, die nicht exakt zu einer solchen Herleitung passen, wie etwa die Stellung von iś in der $i n$-Konstruktion mit nicht-pronominalem Fokus (z. B. $n$ in iś $R^{c} w p r$... „Es ist nicht Re, der herausgekommen ist ..." (CT VII 241k)), wären als sekundäre Weiterentwicklungen einzuschätzen.

So weit ist die Erklärung der Sätze des Typs (1) nicht nur als genetische (oder tiefenstrukturelle) Herleitung dieser Sätze tauglich, sondern auch als eine synchrone Beschreibung ihrer Struktur in historischer Zeit. In anderer Hinsicht ist dagegen ein Abstrich zu machen: Es können solche Sätze mit einer Rang-IV-Erweiterung, einem substantivischen Topic, nach vorn erweitert werden, so daß diese Rang-IV-Erweiterung vor die hypothetische Rang-V-Erweiterung zu stehen kommt:

(22) wis $\boldsymbol{R}^{\boldsymbol{c}} \boldsymbol{w}$ : s:ktt $=f, m$ hn.w igp.t

„Die Barke des Re: Wenn sie fährt, (dann) im Nebel (o. ä.).“ (CT IV 125c S1P)

Es sieht so aus, als ob Sätze des Typs (1), obgleich genetisch aus Rang-V-Erweiterung und Satzkern bestehend, als ganze einen sekundären Satzkern bilden, dem dann nach der Standardregel eine Rang-IV-Erweiterung zugefügt werden kann. Bislang jedenfalls sind abgesehen vom in Rede stehenden Satztyp nur Sätze mit der Abfolge Rang-V-Erweiterung - Rang-IVErweiterung nachgewiesen ${ }^{25} ; z$. B.:

(23) ph.n=k wi: $m=k n \ddot{n} r$ : $r \grave{c} . n \in f,{ }^{n} n h=k$, in $f$ tw $r$ iw pn $n$ (.i) $k 3$

„Wenn du zu mir gekommen bist, so siehe: Gott (und kein anderer) hat veranlaßt, $\mathrm{daß}$ du lebst, dadurch daß (wörtlicher: indem) er dich zu dieser ,Privat'-Insel brachte. ${ }^{\text {c }}$ (Schiffbr. 113f.)

Würde sich im Zuge der weiteren Überprüfung der invertierten Standardtheorie an den Texten herausstellen, daß Rang-V-Erweiterung - verbales Topic (Hintergrund) - und Rang-IVErweiterung - substantivisches Topic - grundsätzlich in beliebiger Weise aufeinanderfolgen dürfen, also, systematisch betrachtet, zu einer Rang-IV/V-Erweiterung, einem allgemeinen unmarkierten Topic, zusammenzufassen sind, so wären die Sätze des Typs (1) auch hinsichtlich der Rang-IV-Erweiterung in der synchronen Beschreibung nicht als Sonderfall einzuschätzen. Sollte sich dagegen im Fortgang der Arbeit keine Revision des wechselseitigen Verhältnisses der RangV- und der Rang-IV-Erweiterung ergeben, blieben Sätze des Typs (1) als ein erstarrtes SpezialSatzmuster stehen, das zwar genetisch aus einem allgemeineren Satzmuster ableitbar ist, in historischer Zeit aber isoliert steht.

${ }^{24}$ Zur hier zugrunde gelegten Interpretation der in-Konstruktion s. die orakelhaften Andeutungen in Tübinger Einführung, Version 1997, \$ 8.5.2, c), Anm. 2 mit anschließender Diskussion; vgl. im übrigen das futurische Pendant ink plus futurisches (prospektives) śčm $=f / i r i(. w)=f$.

${ }^{25}$ Tübinger Einführung, Version 1997, \9.2.1, d) und - das im folgenden zitierte Beispiel - $\int 9.2 .4 .1$. 


\section{Anmerkung 1:}

$\mathrm{Daß}$ das Satzmuster des Typs (1) trotz der hier zunächst einmal angenommenen isolierten Stellung sehr zählebig ist, hängt mit seiner funktionellen Belastung zusammen: Es ist das Standardmittel zur Fokussierung auf Adverbialen. Isolierte Satzmuster sind geradezu typisch für die Fokussierung. Auch die häufige, also wichtige Fokussierung auf das Subjekt wird standardmäßig mit einem solchen bewerkstelligt, nämlich mit der in-Konstruktion. Nur für die weniger häufigen, also weniger wichtigen weiteren Fokussierungen wird ein nicht-spezialisiertes Satzmuster verwandt, der $p w-$ Satz $^{26}$.

\section{Anmerkung 2:}

Selbstverständlich gibt es auch Sätze vom Typ des Satzes (1), in denen außer adverbialen Satzteilen auch noch direkte Objekte vorhanden sind; z. B. $\operatorname{ir} . n t(w)$ $n=i$ htp.t „Mir wurde das Opfer gemacht.“ (CT IV 134c). Auch in diesem Fall kann nur ein adverbialer Satzteil im Fokus stehen (ir.nt(w) $n=i$ htp.t „Mir wurde das Opfer gemacht."), nicht aber ein Objekt wie htp.t „das Opfer" (*ir.nt(w) $n$ i h.tp.t „ „*Das Opfer wurde mir gemacht."). In der Standardtheorie kann das gar nicht anders sein, da nach dieser die betreffenden Sätze einen Adverbialsatz darstellen, in dem eine adverbiale Bestimmung den prädikativen Akzent trägt. Im Rahmen der invertierten Standardtheorie dagegen gibt es von der Syntax her keinen zwingenden Grund dafür, daß nicht auch die Fokussierung auf einen anderen Satzteil als eine adverbiale Bestimmung möglich sein sollte, z. B. auf das Objekt. Es kommt jedoch die Fokussierung auf andere Satzteile deshalb nicht in Betracht, weil sich für die Fokussierung auf Substantive, Subjekt und Objekt, andere Verfahren als Standard durchgesetzt haben, nämlich für die Fokussierung auf Substantive generell der $p w-$ Satz, für die Fokussierung speziell auf das Subjekt die in-Konstruktion. Nicht uninteressant ist hier als argumentative Stütze das Verhältnis zwischen den beiden letztgenannten Konstruktionen. Während der $p w$-Satz grundsätzlich für die Fokussierung auf alle Substantive in Frage kommt und für alle substantivischen Satzteile sogar belegt ist, dominiert bei der Fokussierung auf das Subjekt übermächtig die $i n$-Konstruktion. Hierbei ist noch zu bedenken, daß sich Subjekt und Objekt als Substantive in formaler Hinsicht näher stehen als Substantiv und adverbialer Ausdruck. Man kann es auch so sagen: $\mathrm{Da}$ in den Sätzen vom Typ des Satzes (1) nur auf adverbiale Ausdrücke fokussiert wird, ist eine Frage der Norm, nicht des Systems.

Demgegenüber sind die Probleme der Standardtheorie bei der Erklärung der Sätze des Typs (1), der Erklärung als Adverbialsätze, sehr viel gravierender:

- Der Adverbialsatz kann, anders als der Satz vom Typ (1), nicht mit $n$ - is negiert werden; z. B. richtig:

(24) $n \check{s} m . n=\check{c}$, iś $m(w) t$. $(t) \grave{l}$

„Du bist weggegangen, indem du nicht tot bist.“ (CT VI 380e-f)

dagegen falsch:

(25) *n hn hn.w iś $m$ s:gr

„Die Residenz war nicht in Schweigen.“

(als Negation des im folgenden zitierten korrekten nicht-negierten Satzes hn.w $m$ s:gr „Die Residenz war in Schweigen."

- Umgekehrt kann der Satz vom Typ (1), anders als der Adverbialsatz, nicht mit einer RangIII-Partikel wie iw erweitert werden; z. B. korrekt:

(26) iw: hn.w $m$ s:gr

„Es war der Fall: Die Residenz war in Schweigen." (Sin. R 8)

dagegen falsch:
*iw: iy. $n=i$ 3, $r$ nis $r=k$

„Es war der Fall: Wenn ich hierher kam, (so,) um dich zu rufen.“

(Satz (1), erweitert um iw)

- Schließlich kann ein Umstandssatz als fokussierter adverbialer Ausdruck in Sätzen des Typs (1) stehen, als adverbiales Prädikat im Adverbialsatz dagegen nicht, z. B. korrekt:

(7) $m 33 . t w=f, \boldsymbol{h} 3=f \boldsymbol{r}^{\prime}-p$ ç.t.

„Daß er gesehen wird, (ist,) indem er die Bogentruppe angreift.“

${ }^{26}$ Zur Systematik der Fokussierungskonstruktionen s. Tübinger Einführung, Version 1997, \ 8.5. 
dagegen falsch:

(28) $* h r . t=k,{ }^{c} 3=s$

"Dein Bedarf (ist), indem er groß ist. “

(Satz (3) mit Umstandssatz anstelle des Prädikats $m \operatorname{pr}(. w)=k$,(ist) in deinem Haus“; NB: ,indem er groß ist" ist Prädikat zum Subjekt $\underline{h r . t} k$, „dein Bedarf“; es handelt sich also nicht um hr.t=k: $3=\$$,Dein Bedarf: Er ist groß.“ mit Satzkern $3=\$$, ,ist groß" und topikalisierender Rang-IV-Erweiterung $\underline{h} r . t=k$ „,dein Bedarf“.)

10. Was bislang aus der Standardtheorie vorgeführt wurde, ist in etwa der harte Kern, auf den man alle Vertreter dieser Denkrichtung verpflichten kann. Wer diesen Kern nicht anerkennt, ist kein Standardtheoretiker. Streng genommen ist aber auch kein Standardtheoretiker orthodoxer Observanz, wer nicht noch eine weitergehende Lehre Polotskys vertritt, die Transpositionenlehre. Diese Lehre behauptet, daß die substantivischen und adverbialen Verbalformen, von denen die Rede war - Paradigma (4) -, nicht nur ihrer Funktion nach substantivisch bzw. adverbial sind, nur auch substantivisch bzw. adverbial verwendet werden, sondern daß sie substantivisch bzw. adverbial auch in morphologischer Hinsicht sind, d. h. daß es besondere Flexionsformen des Verbs gibt, von denen einige nur in substantivischer und einige nur in adverbialer Funktion auftreten. Einschränkend sei zugegeben, daß gewisse Aufweichungserscheinungen hinsichtlich der adverbialen Verbalformen auch unter Standardtheoretikern zu beobachten sind, denen man das Bemühen um die orthodoxe Lehre nicht gut absprechen $\mathrm{kann}^{27}$. Man sollte also nicht päpstlicher sein als der Papst. Es sollte aber klargestellt sein, daß zum mindesten Leo Depuydt, als er der Standardtheorie ihren Namen gab ${ }^{28}$, auch diesen Punkt in vollem Umfang als essentielles Merkmal der Standardtheorie sah und darin letzten Endes den Standpunkt vertrat, den Polotsky selbst in seinen letzten krönenden Werken einnahm, in seinen "Transpositions du verbe en égyptien classique" von $1976^{29}$ und in seinen indirekt auch das klassische Ägyptisch betreffenden - „Grundlagen des koptischen Satzbaus“ von 1987 und 1990, seinem großen Schwanengesang ${ }^{30}$.

Jedermann, selbst Polotsky, war bzw. ist klar, daß die so weit formulierte Theorie nicht ausreicht, um alle vorkommenden Sätze mit finiten Verbalformen zu erklären. Es ist ein offenes Geheimnis, daß Polotsky selbst einen großen Bogen um die klassisch-ägyptische Literatur machte, z. B. um Passagen des klassischsten aller klassischen Literaturwerke, der SinuheErzählung, mit vielen śḉm. $n=f$ s in Sequenz, die sich mit den Mitteln der Standardtheorie nicht gerade bequem interpretieren lassen ${ }^{31}$ (auf diese Textstellen wird noch einmal kurz zurückzukommen sein, s. unten $\left.\int 17\right)$. Wie dem im einzelnen auch sei: Es besteht Konsens darüber, daß, wenn man die Standardtheorie als Basis akzeptiert, ein weiterer Ausbau notwendig ist.

11. Es stellt sich jetzt namentlich die Frage, wie man im Rahmen der Standardtheorie die Befunde einordnet, die oben $(\$ 8)$ als Erweiterungen des Satzkerns beschrieben wurden. Zwei Erklärungsstrategien wurden verfolgt. Die eine sucht syntaktische Muster, die sich bei den Kernbefunden der Standardtheorie bewährt haben, auf weitere Befunde zu expandieren. Die zweite hält sich an ältere, vor-standardtheoretische Erklärungsmuster, Erklärungsmuster, die zu Syntagmen etwa in der Art der Satzkernerweiterungen der invertierten Standardtheorie führen.

\footnotetext{
${ }^{27}$ Hier ist namentlich auf Helmut Satzinger zu verweisen, s. H. Satzinger, „The Protasis ir sdm.f ..." - Some Afterthoughts, in: LingAeg 4 (1994), S. $271-274$, bes. S. 274.

${ }^{28}$ L. Depuydt, loc. cit. (s. oben Anm. 3), S. 13-54.

${ }^{29}$ H. J. Polotsky, Les transpositions du verbe en égyptien classique, in: Israel Oriental Studies 6 (1976), S. 1-50 (im folgenden zitiert als "Transpositions").

H. J. Polotsky, Grundlagen des koptischen Satzbaus, Decatur, Georgia 1987-1990, bes. Bd. I, S. 45-140; Bd. II, S. 225-260.

${ }^{31}$ Vgl. A. F. Rainey, The World of Sinuhe, in: Israel Oriental Studies 2 (1972), S. 369-400, bes. S. 371 , Anm. 9.
} 
Auch eine Mischung der aus den alternativen Strategien resultierenden Erklärungen ist möglich. Jede der genannten Satzkernerweiterungen kann möglicherweise unabhängig von jeder anderen unter Berufung auf irgendeines der beiden Konzepte erklärt werden.

Zuerst nun zur Erklärungsstrategie auf der Basis standardtheoretischer Grunderfahrungen. Ein Charakteristikum der Standardtheorie - neben der oben ( $\$ 2$, Anm.) beiläufig angesprochenen Prädikatisierungsstrategie - ist die Verb-Unterordnungsstrategie nach Maßgabe der „verb subjugation hypothesis“, wie Mark Collier eine Grundannahme der Standardtheorie genannt hat ${ }^{32}$. In den behandelten Satzmustern ist das Verb stets in substantivischer oder adverbialer Funktion in einen übergeordneten Satzrahmen eingebettet. Im Satztyp des Satzes (1), der „Emphatischen“ Konstruktion, ist stets eine substantivische Verbalform in der Subjektsstelle eines Adverbialsatzes eingebettet, fallweise auch eine adverbiale Verbalform in der Prädikatsstelle:

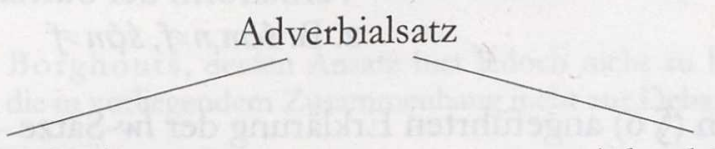

Substantivisches Subjekt, stets eine substantivische Verbalform

Adverbiales Prädikat, evtl. eine adverbiale Verbalform

Im Satztyp des Satzes (5), dem Wechselsatz, sind stets Subjekts- und Prädikatsstelle eines Substantivalsatzes mit substantivischen Verbalformen besetzt:

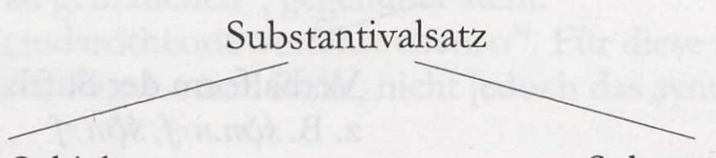

Substantivisches Subjekt, stets eine substantivische Verbalform

Substantivisches Prädikat, stets eine substantivische Verbalform

Im Satztyp des Satzes (2) schließlich, syntaktisch gesehen dem $i w$-Satz, wie man ihn verkürzend nach der typischsten der Partikeln nennen könnte, ist stets eine adverbiale Verbalform eingebettet, etwa als adverbialer Nebensatz (Umstandssatz):

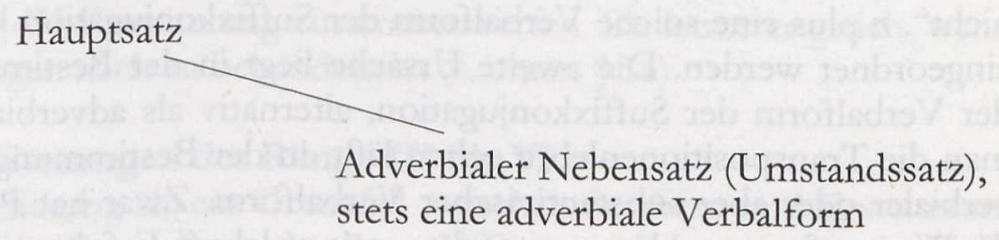

Fast jede der behandelten Satzkernerweiterungen hat man - d. h. nicht jeder jede, sondern der eine oder andere einzelne, aber einer, Friedrich Junge, auch nahezu alle - fast jede der Satzkernerweiterungen hat man versucht als in solcher Weise eingebettet zu erklären.

Der Reihe nach!

\section{Rang-I-Erweiterung}

Die einzige Satzkernerweiterung, die nie als in der genannten Weise eingebettet erklärt worden zu sein scheint, ist die Rang-I-Erweiterung, die Erweiterung z. B. und namentlich durch die Negation $n$, wie sie z. B. in Sätzen vorliegt wie

(32) (in:) $\boldsymbol{n}: \underline{h n n . n=t n}$ „Könnt ihr denn nicht rudern?" (pWestcar 5, 19)

\footnotetext{
${ }^{32}$ M. Collier, Predication and the Circumstantial $s d f m(=f) / s d m . n(\in f)$, LingAeg $2(1992)$, S. $17-65$, bes. S. 19.
} 
(33) $n: w s ̌ b=f n n n n(. i) ~ s r . w(w)$

„Er antwortete nicht diesen Beamten.“ (Bauer B 1, 81 f./neu)

Auf der Linie der Verb-Unterordnungsstrategie könnte man sich Erklärungen analog zu der besprochenen Erklärung der Rang-III-Erweiterung - z. B. mit $i w$ - mit folgender Verbalform der Suffixkonjugation ausdenken. Man könnte versucht sein, die Verbalform nach der Negation $n$ als eingebettet zu erklären, eingebettet in einen Satz $n$ „Es ist nicht der Fall“ o. ä.:

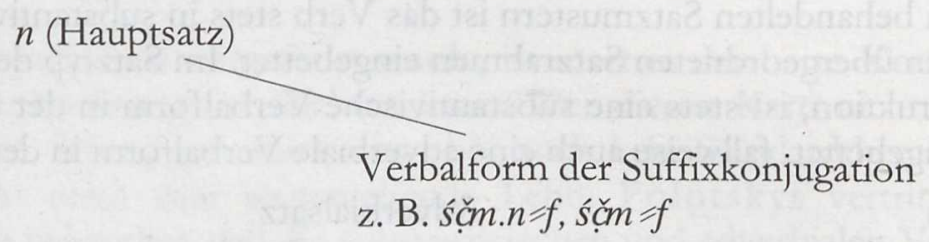

- eine Lösung analog zu der oben (\$ 6 ) angeführten Erklärung der $i w$-Sätze -, oder man könnte versucht sein, die Negation $n$ und die Verbalform der Suffixkonjugation beide in einen Satz einzubetten, $n$ als Subjekt und die Verbalform als Prädikat, analog zu einer Lösung, die Friedrich Junge für die iw-Sätze vorgeschlagen hat (auf die unten bei der Behandlung der Rang-IIIErweiterung noch einzugehen sein wird):

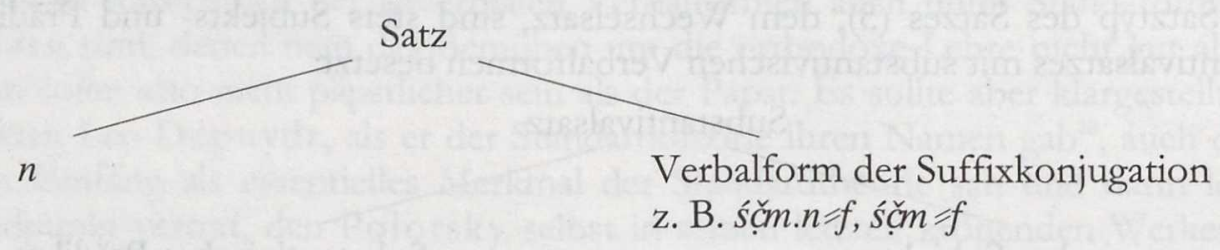

$\mathrm{Daß}$ man von solchen Erklärungen Abstand genommen hat, hat wenigstens zwei Ursachen: Die erste liegt in der sog. Gunnschen Regel, aus der hervorgeht, daß das Tempus einer Verbalform der Suffixkonjugation wie śc̆m. $n=f$ und śḉm $=f$ mit Negation $n$ nicht gleich dem Tempus der gleich (oder wenigstens: in der Regel gleich) aussehenden Verbalform der Suffixkonjugation ohne Negation $n$ ist (z. B. ist śḉm. $n=f$ ohne vorangehende Negation $n$ ein (präsentisches) Perfekt, „er hat gehört", $n$ plus śčm. $n=f$ dagegen ein - negierter - Generalis, „er kann nicht hören, er hört nicht". $n$ plus eine solche Verbalform der Suffixkonjugation kann also nur als Ganzes temporal eingeordnet werden. Die zweite Ursache liegt in der Bestimmung der syntaktischen Funktion der Verbalform der Suffixkonjugation, alternativ als adverbial oder substantivisch bzw., wenn man die Transpositionenlehre gelten läßt, in der Bestimmung der Verbalform als entweder adverbialer oder aber substantivischer Verbalform. Zwar hat Polotsky in den "Transpositions“ die Wortartfrage zu klären versucht - mit welchem Erfolg sei dahingestellt ${ }^{33}$, aber er hat keine weiteren Schlußfolgerungen aus seinem Ergebnis gezogen bezüglich des syntaktischen Baus der Verbindung Negation $n$ plus Verbalform der Suffixkonjugation. Daß er keine Schlußfolgerungen zog, dürfte mit der Heterogenität seiner Teillösungen zusammenhängen: Im Falle der Verbindung der Negation $n$ mit śc̆m. $n=f$ soll die Verbalform eine substantivische sein, im Falle der Verbindung mit śc̆m $=f$ soll jedenfalls keine substantivische vorliegen. Im ersten Fall müßte man den Satz als Substantivalsatz konstruieren:

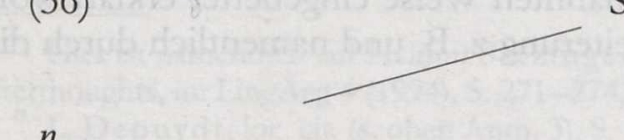

$n_{\text {Subst. }}$

* „Nicht-existierend

${ }^{33}$ Transpositions, S. 44-46.
Satz

(ist), śç̌m. $n=f_{\text {subst. }}$

daß er hört." 
Im zweiten Fall käme, nachdem der Substantivalsatz ausgeschlossen ist, im Rahmen des oben explizierten Kernbereichs der Standardtheorie nur der Ansatz der Verbalform als adverbial in Frage und eine Syntax, bei der $n$ an der Stelle von iw steht, etwa:

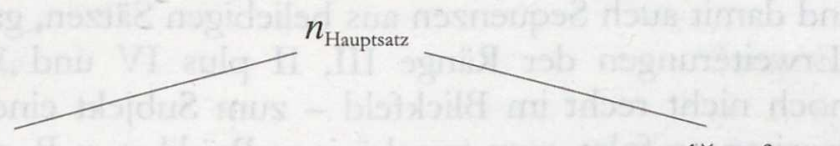

*,Es-ist-nicht-der-Fall,

śc̆m f

Wie gesagt: Polotsky ist nicht so weit gegangen.

\section{Anmerkung:}

Weiter gegangen ist Borghouts, dessen Ansatz hier jedoch nicht zu behandeln ist, da er in eine ganz eigene Theorie eingebettet ist, die in vorliegendem Zusammenhang nicht zur Debatte steht ${ }^{34}$.

Statt dessen wird in der Standardtheorie, um den genannten Problemen - Gunnsche Regel, syntaktische Interpretation - aus dem Wege zu gehen, ohne daß dies unbedingt expressis verbis gesagt wird - die Negation $n$ zum Satzkern geschlagen, d. h. die Verbindung $n$ plus Verbalform der Suffixkonjugation als eine Negativ-Konjugation verstanden, der eine - nicht unbedingt dieselbe - Verbalform der Suffixkonjugation ohne die Negation als "Counterpart", um einen Begriff Sarah Grolls zu gebrauchen ${ }^{35}$, gegenüber steht.

Die invertierte Standardtheorie verfährt ebenso ${ }^{36}$. Für diese spielt jedoch nur das semantische Problem - Gunnsche Regel - eine Rolle, nicht jedoch das syntaktische.

\section{Rang-II-Erweiterung}

An sich käme jetzt der Reihenfolge der Erweiterungen nach die Rang-II-Erweiterung ,an die Reihe“. Man darf sie indes ohne weiteres überschlagen, da diese Erweiterung, die Voranstellung des Subjekts, im Rahmen der Standardtheorie bislang nicht als ein besonderer Fall von den Voranstellungen des Rangs IV unterschieden wird, dem substantivischen Topic, als das beliebige substantivische Ausdrücke stehen können, auch das Subjekt, aber nicht nur das Subjekt. M. a. W.: Wenn man die invertierte Standardtheorie auf die Standardtheorie projiziert, muß man die Rang-II-Erweiterung mit der Rang-IV-Erweiterung zusammenwerfen. Zu letzterer gleich, wenn sie der Reihenfolge der Erweiterungen nach zu behandeln ist (s. unten $₫ 16$ ).

\section{Allgemeines zu den Erweiterungen der Ränge III bis VI}

Nachdem nun die Rang-I-Erweiterung als ein Grenzfall zwischen Satzkern und Erweiterungen zurückgelassen und nachdem die Behandlung der Rang-II-Erweiterung vertagt und auf die Behandlung der Rang-IV-Erweiterung verschoben worden ist, betritt man mit der Rang-IIIErweiterung - $i w$ und dergleichen - das Zentralgebiet der Auseinandersetzungen innerhalb der Standardtheorie und der Auseinandersetzung mit der Standardtheorie, ein Gebiet, das sich von hier über die Rang-IV-Erweiterung - das verbale Topic - bis zur Rang-VI-Erweiterung - den $i r$-Einleitungen (u. dgl.) - erstreckt. Bevor auf die Einzelheiten eingegangen wird, sei ein theoretischer Ansatz herausgestellt, der früheste und zugleich am stärksten generalisierende, der Ansatz Friedrich Junges, den er 1978 in seiner "Syntax der mittelägyptischen Literatursprache“ entwickelte $^{37}$. Dieser Ansatz, ob man ihn nun akzeptiert oder ablehnt, ist schon deshalb von

${ }^{34}$ J. F. Borghouts, Aspectual Values of the Second Tenses in Middle Egyptian, in: BSAK 3 (1988), S. 29-42, bes. S. $33 \mathrm{f}$.

\footnotetext{
${ }^{35}$ S. I. Groll, The Negative Verbal System of Late Egyptian, London-New York 1970.

${ }^{36}$ Tübinger Einführung, Versionen 1994 und 1997, \& 7.3.5 („Rang-I-Komplex-Verbalformen“).

${ }^{37}$ F. Junge, Syntax der mittelägyptischen Literatursprache, Wiesbaden 1978 (im folgenden zitiert als „Syntax“).
} 
Bedeutung, weil er in einer bestimmten wissenschaftsgeschichtlichen Situation Polotskysche Grundannahmen über die Teillösungen Polotskys hinaus so weiterzuentwickeln suchte, daß nicht nur ausgewählte Satztypen erklärt werden konnten, sondern - so zum mindesten die Intention - beliebige Sätze und damit auch Sequenzen aus beliebigen Sätzen, ganze Texte. In dieser Theorie werden alle Erweiterungen der Ränge III, II plus IV und VI - die Rang-VErweiterung war damals noch nicht recht im Blickfeld - zum Subjekt eines Adverbialsatzes, alles, was der jeweiligen Erweiterung folgt, zum zugehörigen Prädikat, z. B. also, was die RangIV-Erweiterung - das substantivische Topic - angeht:

(38)

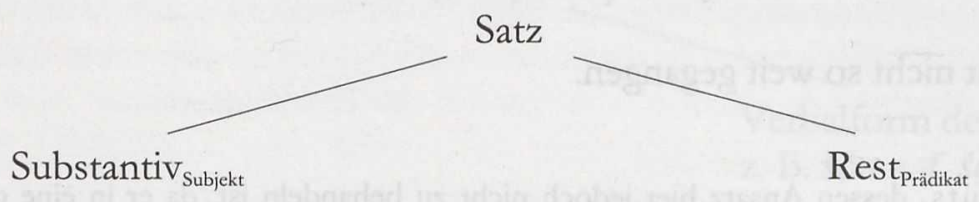

Z. B. ist Satz (10) zu analysieren als:

(39)

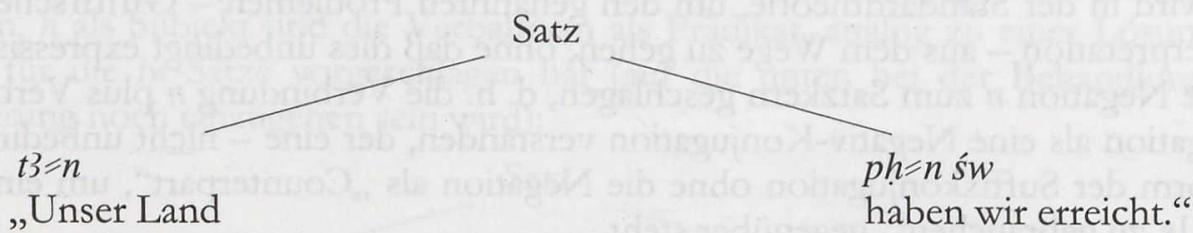

Konsequenterweise wird als „Rest“ in (38) auch wieder ein Satz nach (38) zugelassen, z. B. mit Rang-IV- plus Rang-III-Erweiterung:

(40)

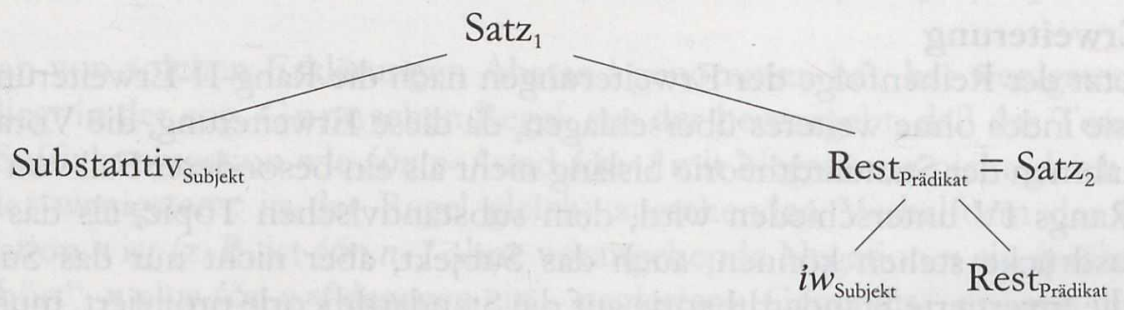

Z.B.:

(41)

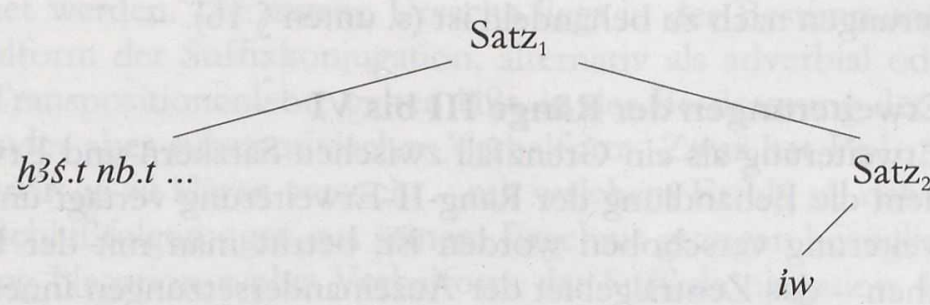

Jedes Fremdland ... (ist, indem) der Fall ist, ir. $n=i h t=i$ im $=s \ldots$

nachdem ich meinen Angriff in ihm machte...

(Sin. B $101 \mathrm{f}$.)

Jetzt aber der Reihe nach die einzelnen Erweiterungen!

\section{Rang-III-Erweiterung}

Hier gibt es die gerade genannte Junge sche Lösung: iw ist Subjekt, der Rest Prädikat:

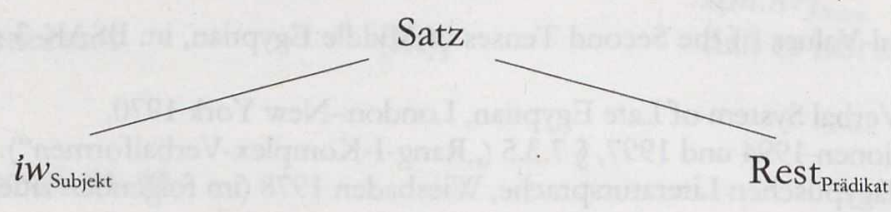


Z. B. ist Satz (9) zu analysieren als:

(43)

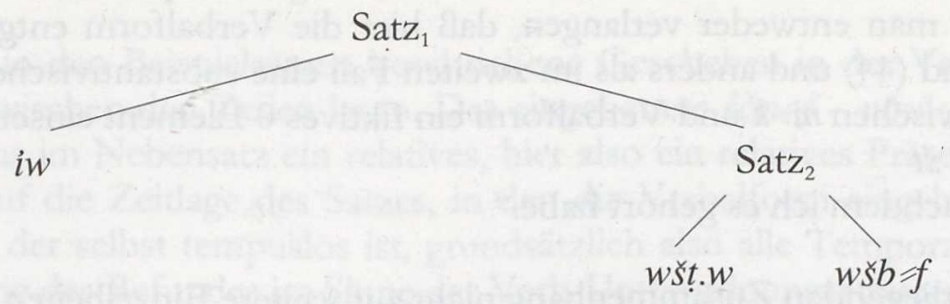

„Der Fall (ist, indem) der Angesprochene antwortet.“

Abgesehen von Junges Göttinger Gefolgschaft - Verf. eingeschlossen, der, beeindruckt durch die grandiose Einfachheit der Jungeschen Globallösung, diese lange Zeit gepredigt hat ${ }^{38}$ -, abgesehen von der Göttinger Gefolgschaft hat die Lösung kaum Anhänger gefunden. Der Hauptgrund dürfte wohl eine semantische Erwägung sein: Sollte man wirklich eine allgemeine Notion wie iw „Der Fall ist“ (o. ä.) zum Gegenstand, zum Subjekt zahlloser Aussagen gemacht haben $^{30}$ ? Statt dessen setzen andere Grammatiker iw und dgl. als Partikel an, mit unterschiedlichen Interpretationen im Detail, evtl. verbunden mit Überlegungen zur Herleitung der Partikel aus einem übergeordneten Satz:

$$
\text { Hauptsatz }
$$

Adverbialer Nebensatz (Umstandssatz), stets eine adverbiale Verbalform

„Der Fall (ist), (indem/nachdem) ...“

Der Haupteinwand gegen jede dieser Erklärungen wendet sich gegen den Ansatz der Verbalform als adverbial, wie sie dies nach dem Paradigma (4) sein muß: Es stehen die Formen der adverbialen Spalte. Setzt man indes an die Stelle der Rang-III-Erweiterung statt einer Rang-IIIPartikel wie iw die rangfreie, illokutive Partikel $m=k$ etc. - die Standardtheorie sieht in dieser Hinsicht zwischen $i w$ und $m=k$ keinen prinzipiellen Unterschied -, so ergibt sich eine Komplikation: Steht $m=k$ vor einem Verbalsatz, so folgt eine Verbalform aus Paradigma (4) entweder direkt auf $m=k$; z. B.:

(45) $m=k$ śc̆m.n $=i$ śt

„Siehe, ich habe es gehört.“ (pBerlin 10016, 2A).

Oder aber es folgt die Verbalform aus Paradigma (4) erst auf ein vorangestelltes Subjekt, eine - im Sinne der invertierten Standardtheorie - Rang-II-Erweiterung; z. B.:

(46) $m=k$ śt śc̆m(.w)

„Siehe, es ist gehört.“ (pBrooklyn 35.1446 B, 13).

Im zweiten Fall, Beispiel (46), handelt es sich bei der Verbalform eindeutig um eine Form aus der adverbialen Spalte des Paradigmas (4), im Beispielfall um das Pseudopartizip. Gleichzeitig ist hier am enklitischen Pronomen eindeutig ablesbar, daß auf $m=k$ ein nominaler und kein adverbialer Ausdruck folgt. Man kann also im Rahmen der Standardtheorie Beispiel (46) entsprechend Modell (44) verstehen als:

„Siehe es, nachdem es gehört worden ist.“

\footnotetext{
${ }^{38}$ Zuletzt, wenigstens noch referierend, in der Tübinger Einführung, Version 1991, \6.4.2.1, Anm. 2.

39. T. Ritter, On Particles in Middle Egyptian, in: LingAeg 2 (1992), S. 127-137 (mit Literaturverweisen).
} 
Verlangt man nach dem Einfachheitskriterium eine analoge Interpretation des ersten Falles, Beispiel (45), so muß man entweder verlangen, daß hier die Verbalform entgegen den Erklärungsmodellen (43) und (44) und anders als im zweiten Fall eine substantivische Verbalform ist, oder aber man muß zwischen $m=k$ und Verbalform ein fiktives 0 -Element einsetzen:

(45) $m=k \emptyset$ śḉm.n =iśt

„Siehe (es), nachdem ich es gehört habe.“

Man braucht im vorliegenden Zusammenhang nicht auf weitere Einzelheiten einzugehen ${ }^{40}$.

Eine einfachere Lösung ist die der invertierten Standardtheorie - und nicht erst dieser -: Es handelt sich bei den Verbalformen einheitlich um verbale Verbalformen, denen $m=k$ und evtl. ein substantivischer Ausdruck (als Objekt zu $m=k$ bzw. Subjekt zur Verbalform) vorangestellt sind:

(45) $m=k: s a c ̌ m . n=i s ́ t$

"Siehe: Ich habe es gehört."

(46) $m=k: s ́ t:$ śç̆m(.w)

„Siehe: Es ist gehört."

\section{Rang-IV-Erweiterung}

Hier gibt es wiederum die bereits illustrierte Jungesche Lösung: Die Rang-IV-Erweiterung das substantivische Topic - ist Subjekt, der Rest Prädikat ${ }^{41}$. Z. B. ist Satz (10) zu analysieren als:

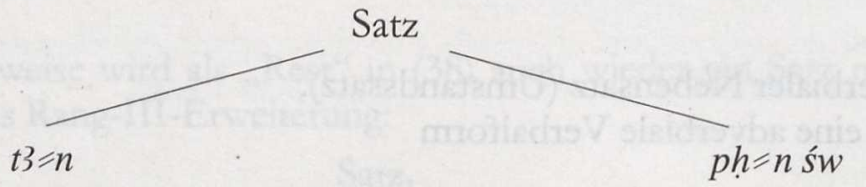

$$
\text { „Unser Land (ist,) nachdem wir es erreicht haben." }
$$

Andere Grammatiker sehen in der Rang-IV-Erweiterung wie die invertierte Standardtheorie ein vor den Satz gestelltes, ein extraponiertes Substantiv. Entscheiden können zwischen den Alternativen sollte man anhand von Sätzen mit der Verbalform śc̆m $=f$ im Satzkern und mit präteritaler Zeitlage, Sätzen in der Art von:

(48) $\ldots . S 3-$ nh.t: $\check{c} t=f:, \ldots, \ldots$

„... Sinuhe sagte: ,..." (Sin. B, 1 f. - nach AOS)

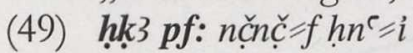

"Jener Herrscher: beriet sich mit mir."

(Sin B, 113f. - die späteren Hss. haben nçnč.n f!)

(10) $t 3=n: p h=n$ św

„Unser Land: haben wir erreicht.“ (Schiffbr. 11)

Soll das, was als Rang-IV-Erweiterung bezeichnet wurde, nicht vor dem Satz stehen, sondern - im Sinne eines erstrebten Ausbaus der Standardtheorie mit ihren originären Mitteln - im Satz,

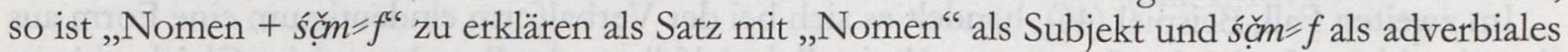
Prädikat. Z. B. ist dann Satz (49) zu verstehen als:

\footnotetext{
${ }^{40}$ Siehe weiter M. Collier, Rez. zu W. Schenkel, Materialien zur Vorlesung Einführung in die klassischägyptische Sprache und Schrift, Wintersemester 1987/88, in: DE 16 (1990), S. 81-88; id., The Circumstantial $\operatorname{s} \underline{d m}(. f) / s \underline{d m} . n(f)$ as Verbal Verb-Forms in Middle Egyptian, in: JEA 76 (1990), S. 73-85; id., Predication and the Circumstantial $s \underline{d} m(=f) / s \underline{d} m . n(=f)$, LingAeg 2 (1992), S. 17-65; id., Circumstantially adverbial? The circumstantial $s \underline{d} m(f) / s \underline{d} . n(f)$ reconsidered, in: S. Quirke (Hg.), Middle Kingdom Studies, New Malden 1991, S. $21-50$.

${ }^{41}$ Junge, Syntax, S. 38 -43; vgl. Tübinger Einführung, Version 1991, § 8.3, d).
} 
"Jener Herrscher (war), indem er sich mit mir beriet."

$\mathrm{Da}$ das hier in den Beispielsätzen beschriebene Geschehen in der Vergangenheit stattfand,

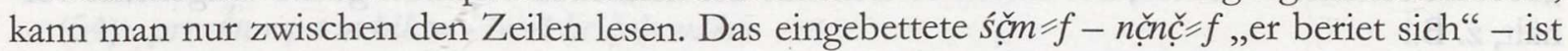
wie jedes Tempus im Nebensatz ein relatives, hier also ein relatives Präsens „,indem er sich beriet", bezogen auf die Zeitlage des Satzes, in den die Verbalform eingebettet ist, hier also des Adverbialsatzes, der selbst tempuslos ist, grundsätzlich also alle Tempora implizieren kann. So weit die Erklärung des Befundes im Sinne der Verb-Unterordnungsstrategie.

Würde man statt nach der Verb-Unterordnungsstrategie der Standardtheorie ein „Nomen + śḉm =f" mit der Verbalform śç̣̆m $f$ und mit präteritaler Zeitlage nach der invertierten Standardtheorie interpretieren, so könnte kein relatives Tempus vorliegen, weil nämlich dann die Verbalform gar nicht eingebettet ist. Die präteritale Zeitlage müßte in der Verbalform selbst liegen. D. h. es könnte sich bei diesem śḉm =f nicht um den Generalis/Aorist aus Paradigma (4) handeln, den die Standardtheorie erwartet, vielmehr müßte es sich um das bislang nicht angesprochene relikthaft im klassischen Ägyptisch noch existierende sog. perfektische šç̣̆ $f$ handeln, ein Vergangenheitstempus, das in der Sprache des Alten Reiches noch in größerem Umfang benutzt wird.

Hier nun kann man den Hebel bei der Entscheidung zwischen den alternativen Erklärungen ansetzen: Man müßte Belege - NB: mit präteritaler Zeitlage - haben, in denen als śḉm $f$ Verben II.gem. oder das Verb r $r \grave{i} i$, geben, veranlassen“ stehen. Bei diesen Verben nämlich und nur bei diesen sind die beiden śç̣̂m $=f s$ in der Schrift voneinander unterscheidbar. Sollte hier die VerbUnterordnung nach der standardtheoretischen Strategie gelten, so sollten die II.gem. geminieren und das Verb $r \check{c} c$, ,geben, veranlassen " die Form $\breve{c}=f$, also ohne anlautendes $r$, zeigen. Sollte dagegen die Erklärung der invertierten Standardtheorie gelten, so sollten die II.gem. nicht

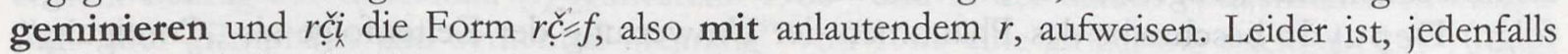
Verf., kein erhellender Beleg bekannt.

\section{Anmerkung:}

Es sei ausdrücklich betont, daß „Nomen“ plus die Verbalform sččm $f$ in Erzählungen nicht selten für die Vergangenheit steht. Daneben kommt nicht selten auch „Nomen“ plus die Verbalform sçcm.n=f, ein Perfekt, vor, das allerdings zumeist nicht als ernsthafter Konkurrent des behandelten „Nomen“ plus sčcm=f in Betracht kommt, sondern eher als Beginn einer „Emphatischen“" Konstruktion verstanden werden muß, dann also - in Begriffen der Standardtheorie - eine substantivische Verbalform ist und nicht die hier allein in Betracht zu ziehende adverbiale. Die Opposition nicht-,,emphatisches" sčm $f$ vs. ,emphatisches" sčm. $n=f$ erinnert an das "Monumental Ramesside“, dessen Befund, wie andernorts skizziert, zeitlich weit über die Ramessidenzeit hinausreicht, nach früher und nach später, also auch im vorliegenden Zusammenhang von Interesse ist ${ }^{42}$.

\section{Rang-V-Erweiterung}

Als letzte Erweiterung sei die Rang-V-Erweiterung genauer betrachtet, das verbale Topic, eine Erweiterung, die für die standardtheoretische Linie, die Verb-Unterordnungsstrategie, ein nicht ganz triviales Problem aufwirft.

Pascal Vernus, der als erster - 1981 - das Problem in aller Schärfe faßte, machte folgende Beobachtung ${ }^{43}$ : Es gibt - „Premier schème“ - „Emphatische“ Konstruktionen mit präteritaler Zeitlage wie z. B.:

(50) hpr.n NN., $n$ hpr.tp.t $n$ hpr.t $t 3$

„Daß NN. entstand, war, bevor der Himmel entstand, bevor die Erde entstand.“ (CT VI 282a-b)

${ }^{42}$ W. Schenkel, „Living in the Past?“, Orientalia 65 (1996), S. 147-156, bes. 152-154.

${ }^{43}$ P. Vernus, Formes „,emphatiques“" en fonction non „emphatique“ dans la protase d'un système corrélatif, in: GM 43 (1981), S. 73-88. 
Das sind Sätze, in denen das an zweiter Stelle, in der Prädikatsstelle, genannte Ereignis vorzeitig ist gegenüber dem an erster Stelle, in der Subjektsstelle, genannten. Daneben gibt es „Second schème“ - Sätze, bei denen es sich mit der zeitlichen Relation genau umgekehrt verhält, z. B.:

(51) hpr.n(=i), hpr.n hpr.t

„Als ich entstanden war, entstand das Entstandene.“ (Turin 54065)

Vernus stellt fest, daß in letzterem Falle der „Nebensatz“ dem „Hauptsatz“ vorausgeht, während es sich in „Emphatischen“ Konstruktionen - mit Verbalform im Prädikat, versteht sich - umgekehrt verhält.

Eine ingeniöse Lösung des Problems, ganz im Sinne der Verb-Unterordnungsstrategie der Standardtheorie, hat Leo Depuydt gefunden ${ }^{44}$. Tatsache ist, daß bei einer derartigen Aufeinanderfolge zweier Vergangenheitstempora vielfach eine rasche Aufeinanderfolge von zwei Ereignissen beschrieben wird. So läßt sich ein Satz wie

(52) h.̣.n t3, ph.n=i Ptn ... (Sin. B 20)

übersetzen mit

„Kaum war es hell geworden, erreichte ich Ptn ...“

„Wörtlich“ heißt der Satz indes, nach dem Muster der „Emphatischen“ Konstruktion verstanden,

„Daß es hell wurde, war, nachdem ich Ptn erreichte."

mit einer offensichtlich inkorrekten Ereignisfolge. (NB: Es handelt sich bei hç.n $t 3$ um die abschnittseinleitende „Tagesformel“, auf die der Bericht über die Geschehnisse eines neuen Tages folgt.) Depuydt beschreibt das Phänomen der Umwertung der Zeitenfolge als „Contiguity“, also etwa ,unmittelbares Aneinandergrenzen“. Die Ereignisse folgen einander so rasch, daß der Eindruck entsteht, das Ergebnis sei bereits vor der Voraussetzung eingetreten. Dementsprechend die sprachliche Formulierung. Ob Vernussens erstes oder zweites Schema vorliegt, ergibt sich aus dem Textzusammenhang. Übrigens sind Sätze von dieser Art z. B. ein guter Teil der Sätze der Sinuhe-Erzählung, von denen oben ( $(10)$ die Rede war.

Es sei als Alternative zu dieser Lösung diejenige der invertierten Standardtheorie zur Disposition gestellt, diejenige als Rang-V-Erweiterung, bei der von vornherein die Zeitenfolge unproblematisch ist; sie entspricht einfach der Folge der Satzteile. Z. B. kann man Satz (50) verstehen als:

„Es wurde hell: (Da) erreichte ich Ptn ...“

Ausdrücklich sei festgehalten, daß Depuydts Lösungsvorschlag überhaupt nur bei präteritaler Zeitlage in Betracht kommt. Zu Sätzen mit anderer Zeitlage s. den folgenden \ 18. Im übrigen sei dahingestellt, ob wirklich die rasche Aufeinanderfolge an all den zahlreichen Textstellen relevant ist, an denen man sie unter Depuydts Hypothese annehmen müßte.

18. Neben den Sätzen mit präteritaler Zeitlage kommen häufig solche mit genereller/aoristischer Zeitlage vor. Hier spielt die zeitliche Reihenfolge keine Rolle. Man könnte solche Sätze also als Wechselsätze der Standardtheorie auffassen. Das geht genau dann gut, wenn die Sätze ordentlich ausbalanciert sind, wie z. B. in:

(53) prr=śn $r$ p.t $m$ bik.w - prr=i $r$ čnh.w $w$ śn

"Steigen sie zum Himmel auf als Falken - steige ich zu ihren Flügeln auf.“ (CT III 24c-25b S1C)

\footnotetext{
${ }^{44}$ L. Depuydt, Conjunction, Contiguity, Contingency, New York - Oxford 1993, S. 117-200.
} 
Aber es gibt, wie Alviero Niccacci in seiner bedeutenden Untersuchung solcher Wechselsätze klar gesehen hat ${ }^{45}$, Sätze ganz ähnlicher Aussage, die nicht sonderlich gut ausbalanciert erscheinen, wie z. B.:

(54) prr=śn r p.t m bik.w - iw=i h̆r čnh.w=śn „Steigen sie zum Himmel auf als Falken - bin ich auf ihren Flügeln.“ (CT III 100h-i S1C)

(55) p3.n NN. m bik-Sbk pw NN. „Flog NN. als Falke auf - war NN. Sobek.“ (CT VI 295 s-t)

Auch hier sei als alternative Lösung die der invertierten Standardtheorie, die Rang-VErweiterung, zur Disposition gestellt, die mit unausbalancierten Sätzen keine Probleme hat:

(54) „Sie steigen zum Himmel auf als Falken: (So) bin ich auf ihren Flügeln.“

(55) „NN. flog als Falke auf: (So) war NN. Sobek.“

Die einheitliche Lösung für präteritale und nicht-präteritale Sätze, wie hier alternativ vorgeschlagen, als Erweiterung plus Satzkern also, ist übrigens nicht erst die Lösung der invertierten Standardtheorie. Es ist eine Lösung, die zumindest Joris F. Borghouts, unbeeinflußt von allen standardtheoretischen Überlegungen und Problemen, gesehen hat ${ }^{46}$.

Nicht dagegen ist die einheitliche Lösung von Friedrich Junge vergleichbar. Es war früher schon ( $($ 14) bemerkt worden, daß Junge das, was in der invertierten Standardtheorie als RangV-Erweiterung bezeichnet ist, in seinem grundlegenden Entwurf einer „Syntax der mittelägyptischen Literatursprache" von 1978 noch nicht im vollen Umfang behandeln konnte, weil die wichtige Beobachtung von Vernus damals noch nicht zur Debatte stand. Er hat dann aber in seinem Beitrag zur Crossroad (I)-Konferenz $1986^{47}$ und in der daraus erwachsenen Monographie über „Emphasis and Sentential Meaning“" von 1989 eine Erklärung nachgeliefert ${ }^{48}$. Junge sieht im Vernusschen Satztyp einen Wechselsatz, somit sieht er, da er auch die Niccaccischen Sätze für Wechselsätze hält, in beiden Fällen den einheitlichen Satztyp Wechselsatz. Damit löst er das temporale Problem. In allen diesen Wechselsätzen entspricht die zeitliche Folge der Folge der Satzglieder. Was aber macht man, wenn Sätze nicht in der Balance sind, wie das eben doch immer wieder einmal, nein: oft, der Fall ist? Nur Borghouts und die invertierte Standardtheorie vermeiden das Problem, da die Balance zwischen Satz-Erweiterung und Satzkern zwar durchaus möglich, zusätzlich möglich ist, nicht jedoch vom Satzbau her gefordert.

\section{Die Rang-VI-Erweiterung}

Man darf sich im gegenwärtigen Zusammenhang eine eingehende Behandlung der Rang-VIErweiterung schenken, die Erweiterung mit ir und dgl. Sie bringt gegenüber dem, was zu den Erweiterungen des Ranges IV und V gesagt wurde, nichts grundsätzlich Neues. Es steht hier Junge gegen den Rest der ägyptologisch-grammatischen Welt: Junge ordnet auch hier unter ${ }^{49}$, andere sehen in traditioneller Weise eine Extraposition, die Voranstellung vor den Satz (bzw. Satzkern).

${ }^{45}$ A. Niccacci, Su una formula dei „Testi dei Sarcofagi“, in: Studium Biblicum Franciscanum, Liber Annuus 30 (1980), S. $197-224$.

${ }^{46}$ J. F. Borghouts, Prominence Constructions and Pragmatic Functions, in: G. Englund/P. J. Frandsen (Hg.), Crossroad, Kopenhagen [1986], S. 45-70, bes. S. 51; vgl. W. Schenkel, Aktuelle Perspektiven der ägyptischen Grammatik, Besprechungsartikel zu Englund/Frandsen, op. cit., in: BiOr 45 (1988), Sp. 269-289, bes. Sp. 276-284.

${ }^{47}$ F. Junge, A Study on Sentential Meaning and the Notion of "Emphasis" in Middle Egyptian, in: Englund/ Frandsen, op. cit. (s. oben Anm. 46), S. 189-254.

${ }^{48}$ F. Junge, Emphasis and Sentential Meaning, Wiesbaden 1989, bes. S. 17. 24-26. 88-94.

49 Junge, Syntax, S. 66-72. 


\section{Die Rang-VII-Erweiterung}

Auch die eingehende Behandlung der Rang-VII-Erweiterung darf man sich im gegenwärtigen Zusammenhang schenken, da sie im Rahmen der Standardtheorie als solche überhaupt nicht in das Blickfeld trat bzw., soweit die betreffenden Fälle behandelt wurden - so die Partikel iśč etc. -, die Rang-VII-Erweiterung als die oben ( $(15)$ behandelte Rang-III-Erweiterung gesehen wurde.

\section{Die rangfreie Erweiterung}

Die rangfreie Erweiterung ist in den Vergleich der Standardtheorie und der invertierten Standardtheorie nur so weit einzubeziehen, als sie fallweise in Konkurrenz zur Rang-III-Erweiterung stehen und diese verdrängen kann: Hierzu ist bereits oben ( $(8)$ das Nötige gesagt worden.

22. Nun könnte man sich am Ende der Gegenüberstellung der Standardtheorie und der invertierten Standardtheorie fragen, welche Theorie denn nun richtig sei, die Standardtheorie oder die invertierte Standardtheorie. Hierzu ist zunächst einmal zu sagen, daß eine Theorie nicht die Realität beschreibt, sondern eine Hypothese über die Realität formuliert, die sich zwar einstweilen bewährt hat, aber immer noch durch neue Beobachtungen zu Fall gebracht werden kann. Was ägyptologische Theorien angeht, so steht zu vermuten, daß sie nicht auf allzu festen Füßen stehen, u. a. einfach deshalb, weil es in einer kleinen Disziplin wie der Ägyptologie stets an Leuten mangelt, die eine Theorie gründlich explorieren und durchtesten. Seit Polotskys ersten Setzungen bastelt man nun schon 50 Jahre lang an der Standardtheorie herum, ohne mit ihrem Ausbau, geschweige denn ihrer Evaluation fertig geworden zu sein. Also ist Vorsicht am Platze, schon gar gegenüber dem, was hier neu als Alternative, als invertierte Standardtheorie, eingebracht wurde.

Was an Sachargumenten aus der Sicht der invertierten Standardtheorie gegen die Standardtheorie spricht, wurde im Laufe der vorangehenden Diskussion bei sich bietender Gelegenheit vorgebracht. Vielleicht darf man unbesorgt so viel behaupten: daß es Punkte in der ägyptischen Grammatik gibt, die die invertierte Standardtheorie besser bewältigt als die Standardtheorie. Es wäre jedoch ein ganz neues Thema, über den unmittelbaren Vergleich ihrer Kernbereiche hinaus Standardtheorie und invertierte Standardtheorie an der Realität der Sprachdaten und/oder an den theoretischen Modellen der allgemeinen Linguistik zu messen und daraus eine umfassende Evaluation der Erklärungsstärke bzw. der theoretischen Akzeptabilität der beiden Theorien zu gewinnen.

Wie immer man sich aber in der Beurteilung der Sachlage entscheiden mag: Wissenschaftsgeschichtlich betrachtet basiert die Standardtheorie auf einem Zweischritt, der nicht naturnotwendig in der Reihenfolge hätte getan werden müssen, in der er tatsächlich getan wurde. Doch was für den Teufel und Gespenster gilt - Goethe, Faust -, gilt auch für die theoriebildenden Wissenschaftler: Wo sie hineinschlüpfen, da müssen sie hinaus. Das erste steht uns frei, beim zweiten sind wir Knechte. Hätte Polotsky seinen zweiten Schritt vor dem ersten getan, gäbe es keine Standardtheorie.

\section{RESÜMEE}

Die Theorie der (Verbal-)Syntax des älteren Ägyptisch, mit deren Entwicklung und Evaluation sich die Grammatiker des Ägyptischen in den letzten Jahrzehnten befaßt haben und der der Status einer Standardtheorie zugesprochen wurde, basiert, wissenschaftsgeschichtlich betrachtet, auf einem Zweischritt. Erster Schritt: die Bestimmung der Funktion der sog. Emphatischen Formen bzw. der mit diesen gebildeten sog. Emphatischen Konstruktion; zweiter Schritt: die Erklärung der übrigen Verbalsätze, u. a. und vor allem des $i w$-Satzes. In diesem Beitrag soll veranschaulicht werden, daß man mit der umgekehrten Schrittfolge zu einer alternativen Theorie gelangen kann, ohne die der Standardtheorie zugrundeliegenden empirischen Befunde - die epochemachenden Entdeckungen H. J. Polotskys - preisgeben zu müssen. Die alternative Theorie, nach der Umkehr der Schrittfolge als Invertierte Standardtheorie bezeichnet, sollte einige Probleme vermeiden, die heute der Standardtheorie angekreidet werden. 